\title{
NOUVELLE
}

\section{Actualités sur la forme intégrée du génome du sixième herpesvirus humain au génome humain (iciHHV-6)}

Agnès Gautheret-Dejean
Service de virologie, APHP, Hôpitaux Universitaires La Pitié Salpêtrière-Charles Foix, Université Paris 6, CIMI Paris, équipe «persistent viral infections », Université Paris Descartes ; Laboratoire de bactériologie et virologie, Faculté de pharmacie, 83, boulevard de l'Hôpital, 75013 Paris, France. agnes.gautheret@aphp.fr
Le sixième herpesvirus humain (HHV-6) Découvert en 1986 chez des sujets atteints de syndromes lymphoprolifératifs et du SIDA (syndrome d'immunodéficience acquise), le sixième herpesvirus humain est un bêtaherpesvirus génétiquement proche du cytomégalovirus humain (CMV), largement répandu dans la population adulte avec une séroprévalence supérieure à $90 \%$ [1]. Deux espèces, HHV-6A et HHV-6B (human herpesvirus-6A, -6B), ont été individualisés en fonction de leurs différences de tropisme, d'épidémiologie et de séquences nucléotidiques [2]. La primo-infection, qui survient principalement durant les trois premières années de vie, est due au HHV-6B. Elle se caractérise par un bref exanthème ${ }^{1}$ maculo-papuleux fébrile, appelé roséole, associé ou non à des convulsions [1]. Le virus reste ensuite latent sous la forme d'un épisome, c'està-dire un génome circularisé, dans le noyau des cellules infectées comme les lymphocytes, les monocytes, certaines cellules épithéliales et, en particulier, les cellules des glandes salivaires [1]. II se réactive de temps à autre, de façon généralement asymptomatique chez les sujets immunocompétents, mais peut provoquer des pathologies redoutables chez les patients immunodéprimés (une encéphalite, un retard à la sortie d'aplasie, une hépatite, des éruptions cutanées fébriles) [1]. Un traitement des atteintes les plus graves est possible, par des médicaments antiviraux comme le ganciclovir, le foscarnet, ou le cidofovir sans qu'il existe à ce jour de recommandations internatio-

\footnotetext{
${ }^{1}$ Un exanthème est une éruption cutanée transitoire.
}

nales [1]. Le diagnostic d'une infection active repose sur la mesure de la charge virale par une amplification génique dans le sang, le liquide céphalo-rachidien ou dans des biopsies (hépatique, digestive ou exceptionnellement cérébrale).

La forme intégrée du HHV-6 (iciHHV-6) Une forme d'infection virale, tout à fait originale chez les herpesvirus humains, a été mise en évidence pour le HHV-6 [1]. Elle avait initialement été découverte, dans les années 1990, par Luppi et al., chez des sujets atteints de syndromes lymphoprolifératifs ou de sclérose en plaque. L'ensemble du génome viral est alors intégré dans le génome humain, plus précisément dans la région subtélomérique de l'un des chromosomes, dans toutes les cellules de l'organisme, y compris les gamètes (Figure 1) [3, 4]. Le mécanisme d'intégration fait intervenir une recombinaison homologue entre les séquences répétées terminales génomiques virales et celles des télomères humains qui leur sont homologues: la séquence $(\mathrm{GGGTTA})_{\mathrm{n}}, \mathrm{n}$ allant de 5 à 83 sur le génome viral, et de 1167 à 1667 sur le génome humain [7]. Wallaschek et al. [5] ont montré que ces répétitions télomériques virales étaient nécessaires pour une intégration efficace du génome viral dans I'ADN chromosomique. La protéine codée par le gène U94 du virus constituerait une intégrase potentielle : elle est en effet homologue de la protéine Rep78/68 de l'adeno-associated parvovirus (AAV) qui est essentielle à l'intégration du matériel génétique de I'AAV dans I'ADN chromosomique [6] $(\rightarrow)$.

$(\rightarrow)$ Voir la Synthèse de A. Rossi et A. Salvetti, $m / s n^{\circ} 2$, février 2016, page 167

La protéine U94 contient d'une part, des domaines liant I'ADN et, d'autre part, des domaines à activités endonucléase, hélicase et ATPase, pouvant intervenir dans l'intégration du HHV-6 [7]. Cependant, pour l'AAV, l'intégration se réalise sur un site précis, alors que pour le HHV-6, elle se fait par recombinaison homologue au niveau des séquences télomériques humaines [6].

L'iciHHV-6, qui concerne les deux espèces HHV-6A et HHV-6B, est observé chez 0,2 à $1 \%$ de la population générale des pays développés $[1,3]$. Il se transmet du parent qui en est porteur, à l'enfant, sur un mode mendélien. D'où le nom actuellement proposé pour cette forme : inherited chromosomally integrated HHV-6, iciHHV-6 [7]. Selon les sujets, les études et les pays, plusieurs chromosomes sont la cible d'une intégration, dont les chromosomes 1, 3, 9, 10, 11, 17, 18, 19 et 22 [3] (et données personnelles). Pour un même sujet, l'intégration s'effectue dans un seul chromosome. La présence de formes intégrées sur deux chromosomes différents chez un même sujet n'a en effet été décrite qu'une seule fois et, compte tenu de la prévalence de l'iciHHV-6, devrait survenir avec une fréquence d'l/10 000 [1].

$\varepsilon n$ dehors de la transmission verticale, l'iciHHV-6 peut également être transmis lors d'une greffe de cellules souches hématopoïétiques lorsque le donneur en est porteur. Après reconstitution de 


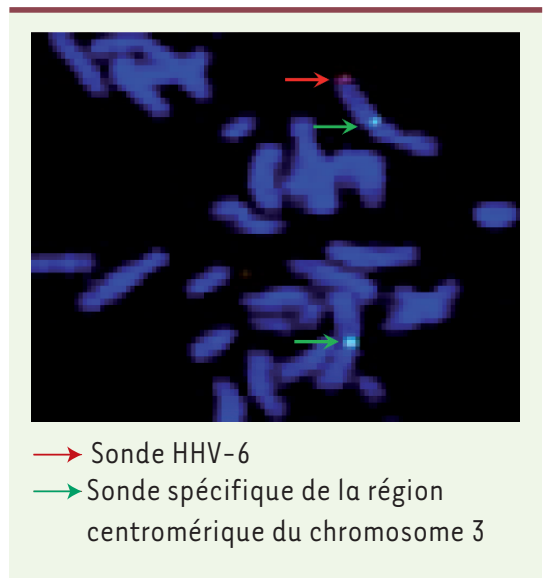

Figure 1. Détection du génome du HHV-6 intégré au génome humain par hybridation in situ en fluorescence. Flèche rouge : sonde HHV-6 ; flèche verte: sonde spécifique de la région centromérique du chromosome 3.

la moelle osseuse, l'ensemble des cellules hématopoïétiques du receveur présentent l'iciHHV-6, ce qui entraîne une charge virale très élevée, supérieure à 1 copie de génome viral par cellule [1]. L'iciHHV-6 soulève de nombreuses questions en termes de lien avec des pathologies, de possibilité de reprise de l'infection active, et pose des problèmes lors du diagnostic et du suivi de l'infection, en particulier chez les patients immunodéprimés [1]. À ce jour, l'iciHHV-6 ne semble pas avoir d'impact majeur sur le développement des enfants qui en sont porteurs et une grande étude canadienne a mis en évidence que la seule pathologie associée à cette forme intégrée est l'angor (angine de poitrine) [8, 9]. Par ailleurs, Hill et al. ont récemment mis en évidence le fait que les patients greffés de cellules souches hématopoïétiques porteurs d'iciHHV-6 avaient deux fois plus de risque de développer une maladie du greffon contre l'hôte aiguë (acute GvHd), et trois fois plus de risque de réactiver le CMV avec une forte virémie [10].
A priori, aucune pathologie marquée par une prolifération cellulaire n'y serait associée. La possibilité d'une réactivation de l'iciHHV-6, avec production de particules virales infectieuses, a cependant été décrite par deux équipes [11, 12]. Gravel et al. ont observé que des femmes enceintes porteuses d'iciHHV-6 pouvaient transmettre à leur fœtus des particules virales dont la séquence génomique était identique à celle de leur forme intégrée [9]. Après réactivation de la multiplication virale, des particules peuvent donc traverser la barrière placentaire et infecter le fœtus. Cette réactivation in vivo a également été démontrée par Endo et al., chez un nourrisson de 2 mois atteint d'un syndrome de déficit immunitaire combiné sévère [12]. Cet enfant, porteur d'un virus iciHHV-6 provenant de son père, présentait de la fièvre, une toux, une diarrhée et un syndrome hémophagocytaire. Du virus réplicatif, dont la séquence génomique était identique à celle de la forme parentale intégrée (analyses réalisées chez l'enfant et son père), était présent dans le sang. La réactivation d'iciHHV-6 chez l'enfant était à l'origine de sa symptomatologie. Le diagnostic formel de présence d'iciHHV-6 est réalisé par la détection ou la quantification du génome du HHV-6 dans les follicules pileux ou les ongles. Une charge sanguine très élevée supérieure à $10^{6}$ copies de génome viral par million de cellules ou par millilitre de sang sur plusieurs prélèvements séquentiels est également un argument fort [1]. À ce jour, il n'existe aucune recommandation de traitement pour prévenir la réactivation de l'iciHHV-6 chez des patients sous traitement immunosuppresseur.

En conclusion, la forme intégrée iciHHV-6 est un phénomène tout à fait original chez les herpesvirus qui est observé chez $1 \%$ de la population générale, sans retentissement majeur sur le développement chez l'enfant, mais qui est associée à l'angine de poitrine. Les questions que HHV-6 soulève ici vont nécessiter de nombreuses années d'études avant d'être résolues. $\diamond$

Latest news on the inherited. chromosomally integrated form of human herpesvirus-6 (iciHHV-6)

\section{LIENS D'INTÉRÊT}

L'auteur déclare participer à des interventions ponctuelles pour les entreprises Biorad et Biosynex.

\section{RÉFÉRENCES}

1. Agut H, Bonnafous P, Gautheret-Dejean A. Laboratory and clinical aspects of human herpesvirus 6 infections. Clin Microbiol Rev 2015 ; 28 : 313-35.

2. Ablashi $D$, Agut $H$, Alvarez-Lafuente R, et al. Classification of HHV-6A and HHV-6B as distinct viruses. Arch Virol $2014 ; 159: 863-70$.

3. Morissette G, Flamand L. Herpesviruses and chromosomal integration. J Virol $2010 ; 84$ : 12100-9.

4. Daibata M, Taguchi T, Nemoto $Y$, et al. Inheritance of chromosomally integrated human herpesvirus 6 DNA. Blood 1999 ; 94 : 1545-9.

5. Wallaschek N, Sanyal A, Pirzer F, et al. The telomeric repeats of human herpesvirus $6 \mathrm{~A}(\mathrm{HHV}-6 \mathrm{~A})$ are required for efficient virus integration. PLoS Pathog 2016 ; 12 : e1005666.

6. Rossi A, Salvetti A. Intégration des vecteurs AAV et mutagenèse insertionnelle. Med Sci (Paris) 2016; 32 : 167-74.

7. Kaufer BB, Flamand L. Chromosomally integrated HHV-6: impact on virus, cell and organismal biology. Curr Opin Virol $2014 ; 9$ : 111-8.

8. Caserta MT, Hall CB, Canfield RL, et al. Early developmental outcomes of children with congenital HHV-6 infection. Pediatrics 2014 ; 134 : 1111-8.

9. Gravel A, Dubuc I, Morissette G, et al. Inherited chromosomally integrated human herpesvirus 6 as a predisposing risk factor for the development of angina pectoris. Proc Natl Acad Sci USA 2015 ; 112 : 8058-63.

10. Hill JA, Magaret AS, Hall-Sedlak R, et al. Outcomes of Hematopoietic Cell Transplantation using Donors or Recipients with Inherited Chromosomally Integrated HHV-6. Blood 2017 June 8. pii: blood-2017-03775759. doi: 10.1182.

11. Gravel A, Hall CB, Flamand L. Sequence analysis of transplacentally acquired human herpesvirus 6 DNA is consistent with transmission of a chromosomally integrated reactivated virus. J Infect Dis 2013 ; 207 : 1585-9.

12. Endo A, Watanabe K, Ohye T, et al. Molecular and virological evidence of viral activation from chromosomally integrated human herpesvirus $6 \mathrm{~A}$ in a patient with $\mathrm{X}$-linked severe combined immunodeficiency. Clin Infect Dis 2014 ; 59 : 545-8.

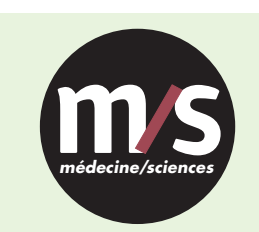

Tarifs d'abonnement $\mathrm{m} / \mathrm{s}-2017$

Abonnez-vous

à médecine/sciences
$>$ Grâce à $\mathrm{m} / \mathrm{s}$, vivez en direct les progrès des sciences biologiques et médicales

Bulletin d'abonnement page 806 dans ce numéro de $\mathrm{m} / \mathrm{s}$

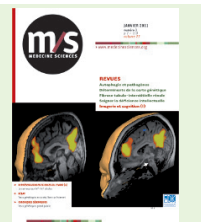

